OPEN ACCESS

Edited by:

Franz Rödel,

University Hospital Frankfurt, Germany

Reviewed by:

Silvia Martina Ferrari,

University of Pisa, Italy

Le Min,

Brigham and Women's Hospital and Harvard Medical School, United States

${ }^{*}$ Correspondence: Hamid Reza Mirzaei h-mirzaei@tums.ac.ir

Specialty section: This article was submitted to

Cancer Immunity and Immunotherapy,

a section of the journal

Frontiers in Oncology

Received: 01 November 2020

Accepted: 02 February 2021

Published: 09 March 2021

Citation:

Karimi A, Alilou S and Mirzaei HR (2021) Adverse Events Following

Administration of Anti-CTLA4 Antibody Ipilimumab.

Front. Oncol. 11:624780. doi: 10.3389/fonc.2021.624780

\section{Adverse Events Following Administration of Anti-CTLA4 Antibody Ipilimumab}

\author{
Amirali Karimi ${ }^{1}$, Sanam Alilou ${ }^{1}$ and Hamid Reza Mirzaei ${ }^{2 *}$ \\ 1 School of Medicine, Tehran University of Medical Sciences, Tehran, Iran, ${ }^{2}$ Department of Medical Immunology, School of \\ Medicine, Tehran University of Medical Sciences, Tehran, Iran
}

Ipilimumab, a monoclonal anti-CTLA4 antibody, paved the path for promising treatments, particularly in advanced forms of numerous cancers like melanoma. By blockading CTLA4, ipilimumab can abolish the higher binding affinity of B7 for CTLA-4, setting CD28 free to act unlimited. This blockade can result in an amplified antitumor immune response, and thereby, boosting more effective tumor regression. However, this blockage can lead to diminished self-tolerance and yielding autoimmune complications. The current review aims to describe adverse events (AEs) following the administration of ipilimumab in different cancers as every benefit comes at a cost. We will also discuss AEs in two different categories, melanoma and non-melanoma, owing to the possible shining promises in treating non-melanoma cancers. As the melanoma settings are more studied than other cancers, it might even help predict the patterns related to the other types of cancers. This similarity also might help physicians to predict adverse events and correctly manage them in non-melanoma cancers using the extensive findings reported in the more-studied melanoma settings. Recognizing the adverse events is vital since most of the adverse events could be reverted while carefully implementing guidelines. Finally, we will also describe the observed effectiveness of ipilimumab in non-melanoma cancers. This effectiveness reveals the importance of understanding the profile of adverse events in this group, even though some have not received FDA approval yet. Further clinical trials and careful systematic reviews may be required to decipher the hidden aspects of therapies with ipilimumab and its related AEs.

Keywords: CTLA4, Anti-CTLA4 antibody, ipilimumab, adverse events, cancer immunotherapy

\section{INTRODUCTION}

Following a tumor antigen presentation by major histocompatibility complex (MHC) class I or II expressed on the antigen-presenting cells (APCs) and a vital second costimulatory signal together can fully promote T cell activation. This signal is transduced through B7-1 (CD80) or B7-2 (CD86) on the APC cell surface upon ligation to CD28 on the T cell surface. CTLA-4 (or CD152), with its superior affinity for B7, could significantly bypass CD28 activation, promote $\mathrm{T}$ cell anergy and inhibit $\mathrm{T}$ cell activation and IL-2 production (1-5). 
Ipilimumab, a monoclonal antibody (MAB) blockading CTLA-4, can abolish the higher binding affinity of B7 for CTLA-4, setting CD28 free to act unlimitedly. This blockade would result in an amplified immune stimulation, boosting tumor annihilation (6). Fortunately, many studies have found that ipilimumab is effective in the treatment of several disorders (7). However, it can be predicted that the blockage of B7: CTLA4 interaction may come out with autoimmune complications, as CTLA-4 signaling plays a critical role in the maintenance of selftolerance to self-antigens $(6,8,9)$.

Ipilimumab increases absolute cell counts of eosinophil, lymphocytes, effector $\mathrm{T}$ cells, and their activation level. On the other hand, it also has a negative impact on some immunosuppressive components of the immune system such as myeloid derivative suppressor cells (MDSC) and regulatory $\mathrm{T}$ cells (10). Therefore, physicians should keep ipilimumab in mind as a possible cause of immune-related adverse events (irAEs). Interestingly, there might be a correlation between higher irAEs and better response to treatment $(11,12)$. The significance of such correlations is still a matter of debate (11, $13,14)$

The current review aims to discuss adverse events (AEs) following the administration of ipilimumab in different cancers, as every benefit comes at a cost (Figure 1). We will discuss AEs in two different categories, melanoma and non- melanoma. As the melanoma setting is more studied than other cancers, it might even help physicians predict the patterns related to the other types of cancers. Furthermore, we will discuss the evidence-based effectiveness of ipilimumab in non-melanoma cancers. This effectiveness reveals the significance of AE profiling this group, even though some have not received FDA approval yet.

\section{EFFECTIVENESS AND ADVERSE EVENTS IN NON-MELANOMA CANCERS}

Some studies have shown that combined treatment with ipilimumab, carboplatin, and etoposide drugs as well as ipilimumab plus nivolumab could benefit patients with advanced small-cell lung cancer (SCLC) and recurrent SCLC patients respectively $(15,16)$. Conversely, two studies have not observed a significant increase in the overall survival or progression-free survival in the patients treated with ipilimumab plus carboplatin and paclitaxel (17), or etoposide and platinum (18) in extensive-disease-SCLC. This was also evident in patients with squamous non-small lung cancer (NSCLC) who were cotreated with ipilimumab and chemotherapeutic drugs (19). However, ipilimumab was found to be highly effective in combination with either nivolumab or carboplatin and paclitaxel

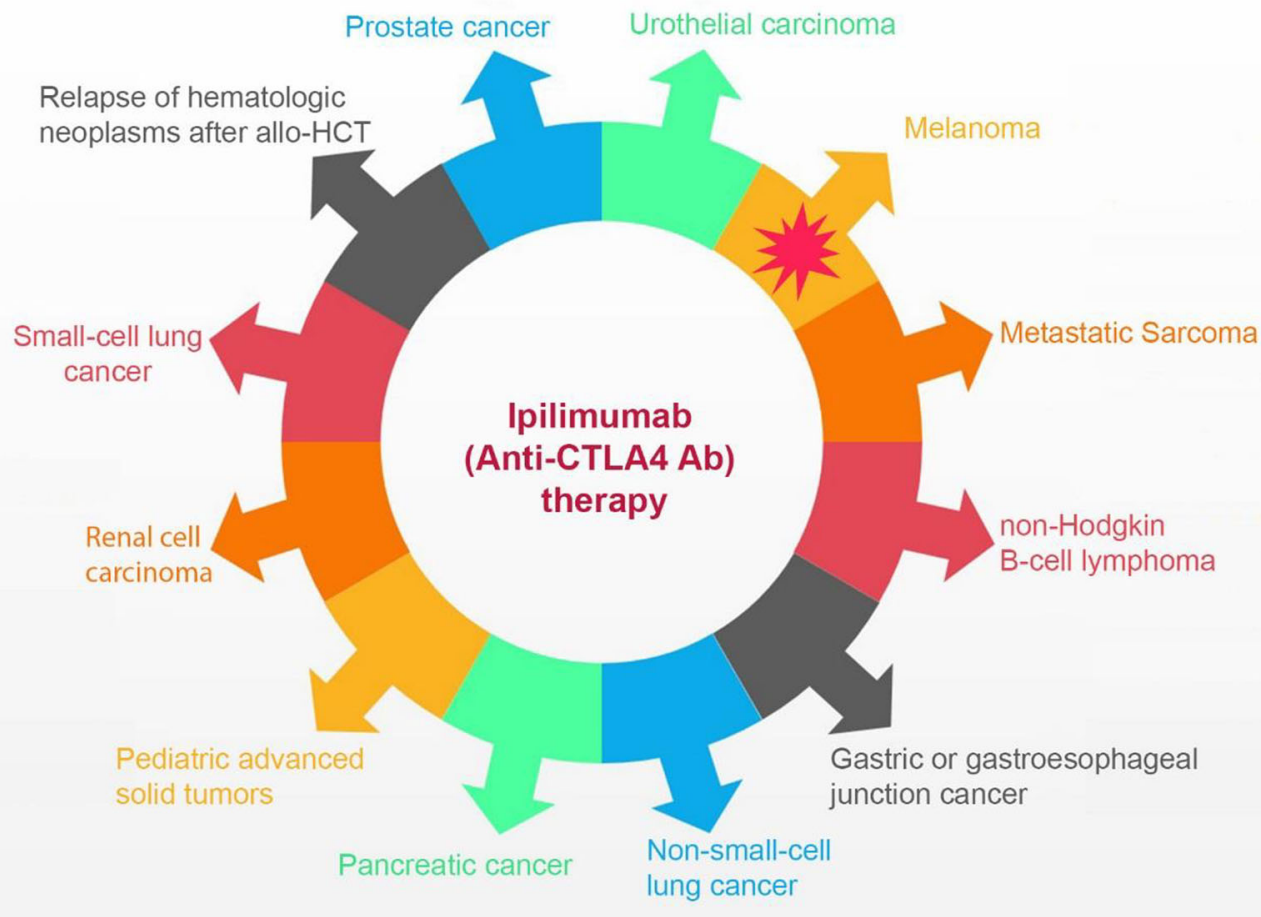

FIGURE 1 | The spectrum of activity of ipilimumab in melanoma and non-melanoma cancers. 
in advanced NSCLC (20-22). The recommended dose of ipilimumab was $10 \mathrm{mg} / \mathrm{kg}$ combined with carboplatin and paclitaxel in Japanese patients with NSCLC (23). In stage II-IIIA NSCLC patients, neoadjuvant use of ipilimumab plus platinum doublet regimen chemotherapy found to be safe and achievable before surgical resection (24).

Ipilimumab was also increased progression-free survival, and prostate-specific antigen (PSA) rates were responded better in the ipilimumab group versus placebo in metastatic castrationresistant prostate cancer (mCRPC) (25-27). Still, they could not prolong overall survival in some studies $(26,27)$. Nevertheless, a dose-escalation clinical trial study has predicted a prolonged overall survival with prostate GVAX/ipilimumab, though its small study population and lack of a control group call its reliability into question (28). Slovin et al. have found a clinical antitumor response following administration of $10 \mathrm{mg} / \mathrm{kg}$ ipilimumab with or without radiotherapy in mCRPC (29). In metastatic urothelial cancer, although chemotherapy plus ipilimumab regime could not improve 1-year overall survival, the regime was feasible and could confer appropriate cytotoxic backbones (30). In a recent study, combination therapy with nivolumab plus ipilimumab could result in high antitumor activity with a manageable safety profile in patients with metastatic urothelial carcinoma (31). Like other types of cancer, treatment with ipilimumab plus nivolumab could improve the overall survival in patients diagnosed with metastatic renal cell carcinoma (mRCC) $(32,33)$. Ipilimumab monotherapy also resulted in some cancer regression cases in $\mathrm{mRCC}$, even in cases with no response to prior immunotherapies (34). In an interesting study, ipilimumab monotherapy was compared to best supportive care (BSC) in metastatic or unresectable locally advanced forms of gastric or gastro esophageal junction tumors. It has been shown that, although ipilimumab monotherapy could not improve immune-related progression-free survival (irPFS), a comparable median overall survival of approximately 1 year and a satisfactory safety profile highlight the significance of the ipilimumab combination therapy in gastric cancer (35). Combination therapy with ipilimumab plus GM-CSF cell-based vaccines (GVAX) could also improve antitumor immune response in the previously treated advanced pancreatic ductal adenocarcinoma (PDA), paving the path for future studies in the field (36). However, ipilimumab alone seems to be ineffective in locally invasive or advanced pancreatic cancer, except for a patient with a significant response, rescuing hope for further attempts and approaches toward immunotherapy, in particular combination immunotherapies (37). Consistent with the above findings, it has been exhibited that single-agent ipilimumab therapy which was implemented on pediatric patients (21 years old or younger) with advanced solid tumors, showed increased survival in a group of patients experiencing irAEs compared to tolerant patients. However, no objective antitumor response was reported (38).

In certain types of sarcoma, ipilimumab plus nivolumab was also found to be promising (39). Ipilimumab has elicited antitumor activity in B-cell non-Hodgkin Lymphoma patients who had relapsed or refractory type of the disease (40).
In the context of recurrence hematologic neoplasms following an allogeneic hematopoietic stem cell transplantation (alloHCT), ipilimumab could be a choice. Still, immune-related complications and graft-versus-host disease (GVHD) occurred in the cases (41). Another study has also established the effectiveness of ipilimumab in the provocation of Graft-versusmalignancy (GVM) following allo-HCT, yet they found no graft rejection or GVHD induced by ipilimumab (42). On the other hand, a specific murine anti-CTLA-4 MAB has been useful in inhibiting chronic GVHD mediated by Th-2 in the mice (43). Table 1 demonstrates a quick review of the non-melanoma clinical trials discussed earlier. By accurately taking prior studies into account and designing further studies, we may cover the drawbacks of ipilimumab monotherapy with the combinatorial therapeutic approaches. It will also help us compare studies and drug regimens and avoid imposing unwanted side effects on the patients.

While embracing the significance of what ipilimumab has brought to cancer immunotherapy, we should note that this immune-checkpoint inhibitor is also associated with side effects. The most common adverse effects related to ipilimumab are the immune-related ones (44). It is noteworthy that many studies on the effectiveness of ipilimumab were primarily designed in the context of malignant melanoma. Therefore, most of the immune-related adverse events (irAEs) discussed in this article occurred in the malignant melanoma setting. Still, some researchers have noted a similar or consistent safety profile in other cancers than melanoma (19, 27, 33, 35). Nevertheless, one study has proposed a favorable safety profile in unresectable locally advanced or metastatic Urothelial Carcinoma (31).

This similarity might help us predict AEs and correctly manage them in non-melanoma cancers, using the several findings reported in the melanoma settings. The findings related to AEs in non-melanoma settings are illustrated in Table 2. The studies observed an increase of $41 \%$ and $48 \%$ in total irAEs where ipilimumab was compared with placebo in monotherapy $(26,27)$ and $17 \%$ and $29 \%$ in the combination with chemotherapy $(18,19)$. However, the total treatment-related AEs were not substantially increased compared to irAEs, as $4 / 9$ of the studies have reported an increase of less than $10 \%$ in this category for the ipilimumab subgroup $(18,19,22,31)$. In the two studies, one of the ipilimumab groups has even demonstrated a lower or equal rate of total AEs compared to placebo or no superior therapeutic effects in the combination therapy groups $(22,31)$.

Nivolumab has been the most promising immune-checkpoint inhibitor in recent years (45). Therefore, we have individually discussed the safety profiles of the studies reporting ipilimumab and nivolumab combination therapies (Table 3). Fatigue (16, 20, $31-33,39,46)$, pruritus $(16,20,21,31-33,46)$, diarrhea $(16,20$, $21,31,33,46)$, rash $(16,21,32,46)$, loss of appetite $(16,32,39)$, and nausea $(20,32)$ were the most common grade 1-2 (G1-2) treatment-related AEs in the ipilimumab and nivolumab combination therapies. Fortunately, most of these conditions did not turn into higher grade AEs and drug discontinuation. 
TABLE 1 | Selected studies evaluating ipilimumab in non-melanoma cancers.

\begin{tabular}{|c|c|c|c|}
\hline Type of cancer & $\begin{array}{l}\text { Study evaluating Ipilimumab } \\
\text { monotherapy }\end{array}$ & $\begin{array}{l}\text { Study evaluating Ipilimumab } \\
\text { combination therapy }\end{array}$ & $\begin{array}{l}\text { Study evaluating Ipilimumab both } \\
\text { alone and combination therapy }\end{array}$ \\
\hline \multirow[t]{2}{*}{ Prostate cancer } & Beer et al. (26) & Jochems et al. (25) & Slovin et al. (29) \\
\hline & Kwon et al. (27) & Santegoets et al. (28) & \\
\hline \multirow[t]{6}{*}{ Non-small-cell lung cancer } & N/A & Govindan et al. (19) & $\mathrm{N} / \mathrm{A}$ \\
\hline & & Hellmann et al. (20) & \\
\hline & & Hellmann et al. (21) & \\
\hline & & Lynch et al. (22) & \\
\hline & & Horinouchi et al. (23) & \\
\hline & & Yang et al. (24) & \\
\hline \multirow[t]{4}{*}{ Small-cell lung cancer } & N/A & Arriola et al. (15) & $\mathrm{N} / \mathrm{A}$ \\
\hline & & Antonia et al. (16) & \\
\hline & & Reck et al. (17) & \\
\hline & & Reck et al. (18) & \\
\hline \multirow[t]{2}{*}{ Renal cell carcinoma } & Yang et al. (34) & Hammers et al. (32) & $\mathrm{N} / \mathrm{A}$ \\
\hline & & Motzer et al. (33) & \\
\hline \multirow[t]{2}{*}{ Urothelial carcinoma } & N/A & Galsky et al. (30) & $\mathrm{N} / \mathrm{A}$ \\
\hline & & Sharma et al. (31) & \\
\hline Pancreatic cancer & Royal et al. (37) & Le et al. (36) & $\mathrm{N} / \mathrm{A}$ \\
\hline Relapse of hematologic neoplasms after & Davids et al. (41) & N/A & $\mathrm{N} / \mathrm{A}$ \\
\hline allo-HCT & Bashey et al. (42) & & \\
\hline Metastatic Sarcoma & D’Angelo et al. (39) & $\mathrm{N} / \mathrm{A}$ & $\mathrm{N} / \mathrm{A}$ \\
\hline non-Hodgkin B-cell lymphoma & Ansell et al. (40) & $\mathrm{N} / \mathrm{A}$ & $\mathrm{N} / \mathrm{A}$ \\
\hline gastric or gastroesophageal junction cancer & Bang et al. (35) & $\mathrm{N} / \mathrm{A}$ & $\mathrm{N} / \mathrm{A}$ \\
\hline Pediatric patients with advanced solid tumors & Merchant et al. (38) & $\mathrm{N} / \mathrm{A}$ & $\mathrm{N} / \mathrm{A}$ \\
\hline
\end{tabular}

Diarrhea $(16,21,31-33,46)$, increased lipase $(16,20,31-33)$, elevated alanine transaminase (ALT) $(31,32,46)$, colitis $(20,31,32)$, rash $(16,21)$, and anemia $(21,39)$ were commonly manifested among G3-4 AEs in patients received these monoclonal antibodies. Amylase was not significantly increased in the patients receiving ipilimumab. In fact, only three subgroups had an elevated amylase on their top 3 G3-4 AEs, all receiving nivolumab $3 \mathrm{mg} / \mathrm{kg}$ (alone, combined with ipilimumab $1 \mathrm{mg} / \mathrm{kg}$, or $3 \mathrm{mg} / \mathrm{kg})(31,32)$. Neither of these studies has reported irAEs.

\section{ADVERSE EFFECTS IN MELANOMA}

In this section, we aim to cover the data published in clinical trials in the advanced melanoma setting. As our knowledge is rapidly increasing, a better understanding of the side effects of ipilimumab will help us to develop more comprehensive safe and effective approaches to manage these complications.

\section{Ipilimumab Monotherapy Versus Placebo}

For two studies discussed in this part, the enrolled patients were at stage III melanoma and were eligible candidates for receiving $10 \mathrm{mg} / \mathrm{kg}$ adjuvant ipilimumab or placebo. Adjuvant ipilimumab or placebo was administered every three weeks for four doses, and then, every three months, for up to three years if a patient received complete treatment. In any case of consent withdrawal, disease progression, high toxicity, or death, the treatment discontinuation was implemented. Eggermont et al. (47) have reported that 465 of 471 (99\%) of the patients in the ipilimumab arm, and 432 of 474 (91\%) of the placebo arm had overall adverse events of any grades, with 254 (54\%) and 118 (25\%) G3-4 adverse events in each group, respectively. Version 3.0 of Common Terminology Criteria for Adverse Events (CTCAE) developed by the National Cancer Institute was utilized to report AEs. Total irAEs were more frequent in the ipilimumab arm than the placebo arm [426 (90.4\%) vs. $183(38.6 \%)]$. The same findings were true for G3-4 irAEs [198 (42.0\%) vs. 12 (2.5\%)]. The most common G3-4 irAEs observed in the ipilimumab arm were gastrointestinal [75 (16\%), 45 patients had diarrhea, and 36 had colitis], hepatic [50 (11\%), half of the patients having increased liver function tests], and endocrine [40 (8\%), 24 cases of hypophysitis and one case of hypothyroidism]. However, in the placebo arm, four patients manifested gastrointestinal complications, one patient hepatic irAEs and no-one demonstrated endocrine irAEs. Dermatological [277 (59\%), pruritus in 176 and rash in 156 patients], gastrointestinal [142 (30\%), mostly diarrhea and colitis], and endocrine [137 (29\%), 62 cases of hypophysitis and 41 cases of hypothyroidism] were the most prevalent G1-2 irAEs in the ipilimumab group. Dermatological irAEs (277 G1-2 vs. 21 G3-4) and hypothyroidism (41 G1-2 vs. 1 G3-4) seemed to have fewer tendencies to severe cases. Drug-related deaths were five (1\%) in ipilimumab $10 \mathrm{mg} / \mathrm{kg}$ and zero in the placebo group. Causes of the deaths related to treatment in the ipilimumab $10 \mathrm{mg} / \mathrm{kg}$ subgroup were colitis in three cases (two with gastrointestinal perforation), and myocarditis and multiorgan failure with Guillen-Barre syndrome each caused one death (47). A study conducted by Coens et al. (48) on 951 stage III cutaneous melanoma patients have demonstrated that ipilimumab could not lead to a clinically relevant decline in global health-related quality of life (HRQoL) compared with placebo. 
TABLE 2 | Summary of adverse events in non-melanoma cancers in clinical trials possessing ipilimumab group(s) of more than 50 patients and reporting safety profile.

ID Study Population for safety 1. Mean/Median age $2 . \quad$ Target cancer analysis

Female (\%)

Dosage and concurrent

Incidence of total

Incidence of

total irAEs

Claiming a similarand/or

Ipilimum

AEs $^{*}(\%)$

(\%)

consistent pattern to

1 (26) 1) $n=399$ (lpi)

2) $n=199$ (placebo)

mCRPC

$4 \mathrm{D}$ of $10 \mathrm{mg} / \mathrm{kg}$ E3M

1) $82 \%$

1) $77 \%$

2) 69

2) $49 \%$

1) $77 \%$

melanoma settings

2 (27) 1) $n=393$ (lpi)

$2.0 \%$ in both
1. 1) 69

2) $n=396$ (placebo)

$3 \begin{aligned} \text { 1) } n=388(\text { (lpi + chemo) } \\ \text { 2) } n=361 \text { (placebo + chem })\end{aligned}$

$$
\text { 2) } 67.5
$$

mCRPC

4D of $10 \mathrm{mg} / \mathrm{kg}$ E3W

1) $75 \%$

1) $63 \%$

$\mathrm{N} / \mathrm{A}$

2. $0 \%$ in both 2) $n=361$ (placebo + chemo)

1.64 in both

2. 1) $16 \%$

Advanced squamous NSCLC

$4 \mathrm{D}$ of $10 \mathrm{mg} / \mathrm{kg}$ E3W +

Paclitaxel and Carboplatin

2) $45 \%$

2) $22 \%$

1) $89 \%$

1) $69 \%$

2) $52 \%$

1) $75.2 \%$

$\mathrm{N} / \mathrm{A}^{+}$

Nivolumab $3 \mathrm{mg} / \mathrm{kg}$

$$
\text { 2) } n=391 \text { (Nivol) }
$$

N/A (available for high

Stage IV or recurrent NSCLC not $4 \mathrm{D}$ of $1 \mathrm{mg} / \mathrm{kg}$ E6W

previously treated with

2) $64.2 \%$

subgroup) chemotherapy

5 (22) 1) $\mathrm{n}=71$ (concurrent Ipi)

2) $n=67$ (phased Ipi)

1. 1) 59

Stage IIIB/IV or recurrent NSCLC

Concurrent Ipi: chemo + 4D

1) $76 \%$

2) $82 \%$

3) $n=65$ (placebo + chemo)

2. 1) $24 \%$

2) $28 \%$

3) $26 \%$

6 (16) 1) $\mathrm{n}=54($ (Ipi1 + Nivol3)

2) $n=98$ (Nivol3)

3) $n=61$ (Ipi3 + Nivol1)

1. 1) 61

of lpi followed by 2D of

3) $80 \%$

placebo E3W

chemo $+4 \mathrm{D}$ of placebo

followed by 2D of Ipi E3W

Recurrent SCLC

4D of $1 \mathrm{mg} / \mathrm{kg}$ lpi E3W +

1) $74 \%$

2) $53 \%$

$\begin{array}{ll}\text { Nivolumab } 3 \mathrm{mg} / \mathrm{kg} & \text { 2) } 53 \% \\ & \text { 3) } 79 \%\end{array}$

2. 1) $22 \%$

2) $37 \%$

3) $26 \%$

$7 \quad(18) \quad$ 1) $n=478($ (lpi + chemo $)$

1. 1) 62

2) $n=476$ (placebo + chemo)

2) 63

2. 1) $34 \%$

2) $32 \%$

$8^{\mathrm{a}} \quad$ (33) 1$) \mathrm{n}=547(\mathrm{lpi}+\mathrm{Nivol})$

2) $n=535$ (Sunitinib)

1. both 62

2. 1) $25 \%$

2) $28 \%$

9 (31) 1) $\mathrm{n}=92($ (Ipi3 + Nivol1)

2) $n=104($ (lpi1 + Nivol3)

3) $n=78$ (Nivol3)

1. 1) 64.0

2) 63.0

3) 65.5

2. 1) $26 \%$

2) $19 \%$

3) $46 \%$

10 (35)

1) $n=57$ (Ipi)

2) $n=57$ (Best supportive

care)

2) 62

Extensive-stage SCLC

4D of $10 \mathrm{mg} / \mathrm{kg}$ E3W +

Etoposide and Platinum

1) $82 \%$

2) $76 \%$

1) $57 \%$

2) $28 \%$

Advanced RCC

$4 \mathrm{D}$ of $3 \mathrm{mg} / \mathrm{kg}$ E3W +

1) $93 \%$

$\mathrm{N} / \mathrm{A}^{\dagger}$

2) $97 \%$

$\mathrm{N} / \mathrm{A}^{+}$

Unresectable locally advanced or 1) 4D of $3 \mathrm{mg} / \mathrm{kg}$ E3W +

Nivolumab $3 \mathrm{mg} / \mathrm{kg}$

1) $80.4 \%$

2) $84.6 \%$

2) $4 \mathrm{D}$ of $1 \mathrm{mg} / \mathrm{kg}$ E3W +

3) $84.6 \%$

$N / A^{\dagger}$

Nivolumab $3 \mathrm{mg} / \mathrm{kg}$

1) $71.9 \%$

2) $55.6 \%$

Metastatic Gastric or GEJ

Cancer

*AE, Adverse events; irAE, Immune-related adverse events; $M C R P C$, metastatic Castration-Resistant Prostate Cancer; 4D, Four Doses; E3M, Every three months; E3W, Every three weeks; NSCLC, Non-Small-cell lung cancer; SCLC, Smallcell lung cancer; RCC, Renal Cell Carcinoma; UC, Urothelial Carcinoma; GEJ, Gastroesophageal cancer. ${ }^{\dagger}$ N/A, Not applicable due to no claims by the authors.

${ }^{a}$ This study does not compare ipilimumab to placebo. 


\begin{tabular}{|c|c|c|c|c|c|c|c|}
\hline Study & $\begin{array}{l}\text { Population for safety } \\
\text { analysis }\end{array}$ & $\begin{array}{c}\text { 1. Mean/Median age } 2 . \\
\text { Female }(\%)\end{array}$ & Target cancer & $\begin{array}{c}\text { Incidence of total } \\
\text { treatment-related } \mathrm{AEs}^{\star}\end{array}$ & $\begin{array}{l}\text { Incidence of } \\
\text { G3-4 AEs }\end{array}$ & 3 most common G1-2 AEs & 3 most common G3-4 AEs \\
\hline (16) & $\begin{array}{l}\text { 1) } n=54(\text { (lpi } 1 \mathrm{mg} / \mathrm{kg}+\text { Nivol } \\
3 \mathrm{mg} / \mathrm{kg}) \\
\text { 2) } n=61(\text { (lpi } 3 \mathrm{mg} / \mathrm{kg}+\text { Nivol } \\
1 \mathrm{mg} / \mathrm{kg}) \\
\text { 3) } n=98(\text { Nivol } 3 \mathrm{mg} / \mathrm{kg})\end{array}$ & $\begin{array}{l}\text { 1. 1) } 61 \\
\text { 2) } 66 \\
\text { 3) } 63 \\
\text { 2. 1) } 22 \% \\
\text { 2) } 26 \% \\
\text { 3) } 37 \%\end{array}$ & Recurrent SCLC & $\begin{array}{l}\text { 1) } 40(74 \%) \\
\text { 2) } 48(79 \%) \\
\text { 3) } 52(53 \%)\end{array}$ & $\begin{array}{l}\text { 1) } 10(19 \%) \\
\text { 2) } 18(30 \%) \\
\text { 3) } 13(13 \%)\end{array}$ & $\begin{array}{l}\text { 1) 1. Fatigue: } 12(22 \%) \\
\text { 2. Diarrhea: } 8 \text { (15\%) } \\
\text { 3. Decreased appetite: } 6 \\
\text { (11\%) } \\
\text { 2) 1. Fatigue: } 16(26 \%) \\
\text { 2. Pruritus: } 11 \text { (18\%) } \\
\text { 3. Diarrhea \& Rash: } 10(16 \%) \\
\text { 3) 1. Pruritus: } 11(11 \%) \\
\text { 2. Fatigue: } 10 \text { (10\%) } \\
\text { 3. Diarrhea \& Nausea: } 7 \text { (7\%) }\end{array}$ & $\begin{array}{l}\text { 1) 1. Dyspnea: } 2 \text { (4\%) } \\
\text { 2. Several others: } 1(2 \%) \\
\text { 2) 1. Increased lipase: } 5(8 \%) \\
\text { 2. Diarrhea: } 3(5 \%) \\
\text { 3. Rash (maculopapular): } 2(3 \%) \\
\text { 3) 1. Several AEs: } 1(1 \%)\end{array}$ \\
\hline (20) & $\begin{array}{l}\text { 1) } \mathrm{n}=39(\mathrm{lpi} 1 \mathrm{mg} / \mathrm{kg} \text { E6W + } \\
\text { Nivol } 3 \mathrm{mg} / \mathrm{kg}) \\
\text { 2) } \mathrm{n}=38(\mathrm{lpi} 1 \mathrm{mg} / \mathrm{kg} \text { E12W } \\
+ \text { Nivol } 3 \mathrm{mg} / \mathrm{kg})\end{array}$ & $\begin{array}{l}\text { 1. 1) } 62 \\
\text { 2) } 68 \\
\text { 2. 1) } 38 \% \\
\text { 2) } 55 \%\end{array}$ & $\begin{array}{l}\text { Recurrent stage Illb or } \\
\text { stage IV, chemotherapy- } \\
\text { naive NSCLC }\end{array}$ & $\begin{array}{l}\text { 1) } 28(72 \%) \\
\text { 2) } 31(82 \%)\end{array}$ & $\begin{array}{l}\text { 1) } 13(33 \%) \\
\text { 2) } 14(37 \%)\end{array}$ & $\begin{array}{l}\text { 1) } 1 \text { \& 2. Diarrhea \& Fatigue: } \\
8 \text { (21\%) } \\
\text { 3. Pruritus \& Nausea: } 5(13 \%) \\
\text { 2) 1. Pruritus: } 9(24 \%) \\
\text { 2. Diarrhea: } 7(18 \%) \\
\text { 3. Nausea: } 6(16 \%)\end{array}$ & $\begin{array}{l}\text { 1) } 1 \text { \& 2. Adrenal insufficiency \& Colitis: } \\
2 \text { (5\%) } \\
\text { 3. Several others: } 1 \text { (3\%) } \\
\text { 2) 1. Increased lipase: } 3(8 \%) \\
\text { 2. Pneumonitis: } 2(5 \%) \\
\text { 3. Several others: } 1(3 \%)\end{array}$ \\
\hline$(21)^{a}$ & $\begin{array}{l}\text { 1) } n=576 \text { (lpi } 1 \text { mg/kg + } \\
\text { Nivol } 3 \text { mg/kg) } \\
\text { 2) } n=391 \text { (Nivol } 240 \text { mg) } \\
\text { 3) } n=570 \text { Chemotherapy }\end{array}$ & $\begin{array}{l}\text { N/A (available for high tumor } \\
\text { mutational burden subgroup) }\end{array}$ & $\begin{array}{l}\text { Stage IV or recurrent } \\
\text { NSCLC not previously } \\
\text { treated with } \\
\text { chemotherapy }\end{array}$ & $\begin{array}{l}\text { 1) } 433(75.2 \%) \\
\text { 2) } 251(64.2 \%) \\
\text { 3) } 460(80.7 \%)\end{array}$ & $\begin{array}{l}\text { 1) } 180 \\
(31.2 \%) \\
\text { 2) } 74 \\
(18.9 \%) \\
\text { 3) } 206 \\
(36.1 \%)\end{array}$ & $\begin{array}{l}\text { 1) 1. Rash: } 87(15.1 \%) \\
\text { 2. Diarrhea: } 85(14.8 \%) \\
\text { 3. Pruritus: } 78 \text { (13.5\%) } \\
\text { 2) } 1 \text { \& 2. Diarrhea \& Fatigue: } \\
\text { 41 (10.5\%) } \\
\text { 3. Rash: } 40 \text { (10.2\%) }\end{array}$ & $\begin{array}{l}\text { 1) } 1,2 \text { \& 3. Anemia, Diarrhea, \& Rash: } \\
\text { 9 (1.6\%) } \\
\text { 2) } 1 \text { \& 2. Diarrhea \& Rash: } 3(0.8 \%) \\
\text { 3. Anemia, Asthenia, \& Fatigue: } 2 \\
\text { (0.5\%) }\end{array}$ \\
\hline (31) & $\begin{array}{l}\text { 1) } \mathrm{n}=92(\text { (Ipi } 3 \mathrm{mg} / \mathrm{kg}+\text { Nivol } \\
1 \mathrm{mg} / \mathrm{kg}) \\
\text { 2) } \mathrm{n}=104(\mathrm{Ipi} 1 \mathrm{mg} / \mathrm{kg}+ \\
\text { Nivol } 3 \mathrm{mg} / \mathrm{kg}) \\
\text { 3) } \mathrm{n}=78(\text { Nivol } 3 \mathrm{mg} / \mathrm{kg})\end{array}$ & $\begin{array}{l}\text { 1. 1) } 64.0 \\
\text { 2) } 63.0 \\
\text { 3) } 65.5 \\
\text { 2. 1) } 26 \% \\
\text { 2) } 19 \% \\
\text { 3) } 46 \%\end{array}$ & $\begin{array}{l}\text { Platinum-pretreated } \\
\text { unresectable locally } \\
\text { advanced or metastatic } \\
\text { UC }\end{array}$ & $\begin{array}{l}\text { 1) } 74(80.4 \%) \\
\text { 2) } 88(84.6 \%) \\
\text { 3) } 66(84.6 \%)\end{array}$ & $\begin{array}{l}\text { 1) } 36 \\
(39.1 \%) \\
\text { 2) } 32 \\
(30.8 \%) \\
\text { 3) } 21 \\
(26.9 \%)\end{array}$ & $\begin{array}{l}\text { 1) 1. Pruritus: } 29(31.5 \%) \\
\text { 2. Fatigue: } 24(26.1 \%) \\
\text { 3. Diarrhea: } 21(22.8 \%) \\
\text { 2) 1. Fatigue: } 30(28.8 \%) \\
\text { 2. Pruritus: } 28(26.9 \%) \\
\text { 3. Diarrhea: } 19(18.3 \%) \\
\text { 3) } 1 \text { \& } 2 \text {. Fatigue \& Pruritus: } \\
26(33.3 \%) \\
\text { 3. Maculopapular Rash: } 14 \\
\text { (17.9\%) }\end{array}$ & $\begin{array}{l}\text { 1) 1. Diarrhea: } 9 \text { (9.8\%) } \\
\text { 2. Elevated ALT: } 6(6.5 \%) \\
\text { 3. Elevated lipase: } 4(4.3 \%) \\
\text { 2) } 1 \text { \& 2. Elevated ALT \& lipase: } 6 \\
\text { (5.8\%) } \\
\text { 3. Diarrhea: } 5 \text { (4.8\%) } \\
\text { 3) 1. Elevated lipase: } 5(6.4 \%) \\
\text { 2. Elevated amylase: } 4(5.1 \%) \\
\text { 3. Maculopapular Rash: } 3(3.8 \%)\end{array}$ \\
\hline (32) & $\begin{array}{l}\text { 1) } n=6 \text { (Ipi } 3 \mathrm{mg} / \mathrm{kg}+\text { Nivol } \\
3 \mathrm{mg} / \mathrm{kg}) \\
\text { 2) } \mathrm{n}=47 \text { (Ipi } 3 \mathrm{mg} / \mathrm{kg}+\text { Nivol } \\
1 \mathrm{mg} / \mathrm{kg}) \\
\text { 3) } n=47 \text { (Ipi } 1 \mathrm{mg} / \mathrm{kg}+\text { Nivol } \\
3 \mathrm{mg} / \mathrm{kg})\end{array}$ & $\begin{array}{l}\text { 1. 1) } 54.8 \\
\text { 2) } 55.6 \\
\text { 3) } 53 \\
\text { 2. 1) } 16.7 \\
\text { 2) } 23.4 \\
\text { 3) } 8.5\end{array}$ & Metastatic RCC & $\begin{array}{l}\text { 1) } 6(100 \%) \\
\text { 2) } 45(95.7 \%) \\
\text { 3) } 43(91.5 \%)\end{array}$ & $\begin{array}{l}\text { 1) } 5(83.3 \%) \\
\text { 2) } 29 \\
\text { (61.7\%) } \\
\text { 3) } 18 \\
(38.3 \%)\end{array}$ & $\begin{array}{l}\text { 1) 1. Fatigue: } 6(100 \%) \\
\text { 2. Hypothyroidism: } 5 \text { ( } 83.3 \%) \\
\text { 3. Arthralgia \& Decreased } \\
\text { appetite: } 4(66.7 \%) \\
\text { 2) 1. Fatigue: } 29(61.7 \%) \\
\text { 2. Nausea: } 21(44.7 \%) \\
\text { 3. Pruritus: } 17(36.2 \%) \\
\text { 3) 1. Fatigue: } 24(51.1 \%) \\
\text { 2 \& 3. Pruritus \& Rash: } 15 \\
\text { (31.9\%) }\end{array}$ & $\begin{array}{l}\text { 1) } 1,2 \text {, \& } 3 \text {. Headache, Increased } \\
\text { amylase, \& Increased lipase: } 2(33.3 \%) \\
\text { 2) 1. Increased lipase: } 13(27.7 \%) \\
\text { 2. Increased ALT: } 10 \text { (21.3\%) } \\
\text { 3. Diarrhea \& Colitis: } 7 \text { (14.9\%) } \\
\text { 3) 1. Increased lipase: } 7 \text { (14.9\%) } \\
\text { 2 \& 3. Diarrhea, Pyrexia, Increased } \\
\text { ALT, AST, \& amylase: } 2 \text { (4.3\%) }\end{array}$ \\
\hline$(33)^{a}$ & $\begin{array}{l}\text { 1) } n=547 \text { (lpi } 3 \mathrm{mg} / \mathrm{kg}+ \\
\text { Nivol } 3 \mathrm{mg} / \mathrm{kg}) \\
\text { 2) } \mathrm{n}=535 \text { (Sunitinib) }\end{array}$ & $\begin{array}{l}\text { 1. both } 62 \\
\text { 2. 1) } 25 \% \\
\text { 2) } 28 \%\end{array}$ & $\begin{array}{l}\text { Previously untreated } \\
\text { advanced RCC }\end{array}$ & $\begin{array}{l}\text { 1) } 509(93 \%) \\
\text { 2) } 521(97 \%)\end{array}$ & $\begin{array}{l}\text { 1) } 250(46 \%) \\
\text { 2) } 335(63 \%)\end{array}$ & $\begin{array}{l}\text { 1) 1. Fatigue: } 179(33 \%) \\
\text { 2. Pruritus: } 151(28 \%) \\
\text { 3. Diarrhea: } 124(23 \%)\end{array}$ & $\begin{array}{l}\text { 1) 1. Increased lipase: } 56(10 \%) \\
\text { 2. Fatigue: } 23(4 \%) \\
\text { 3. Diarrhea: } 21(4 \%)\end{array}$ \\
\hline
\end{tabular}




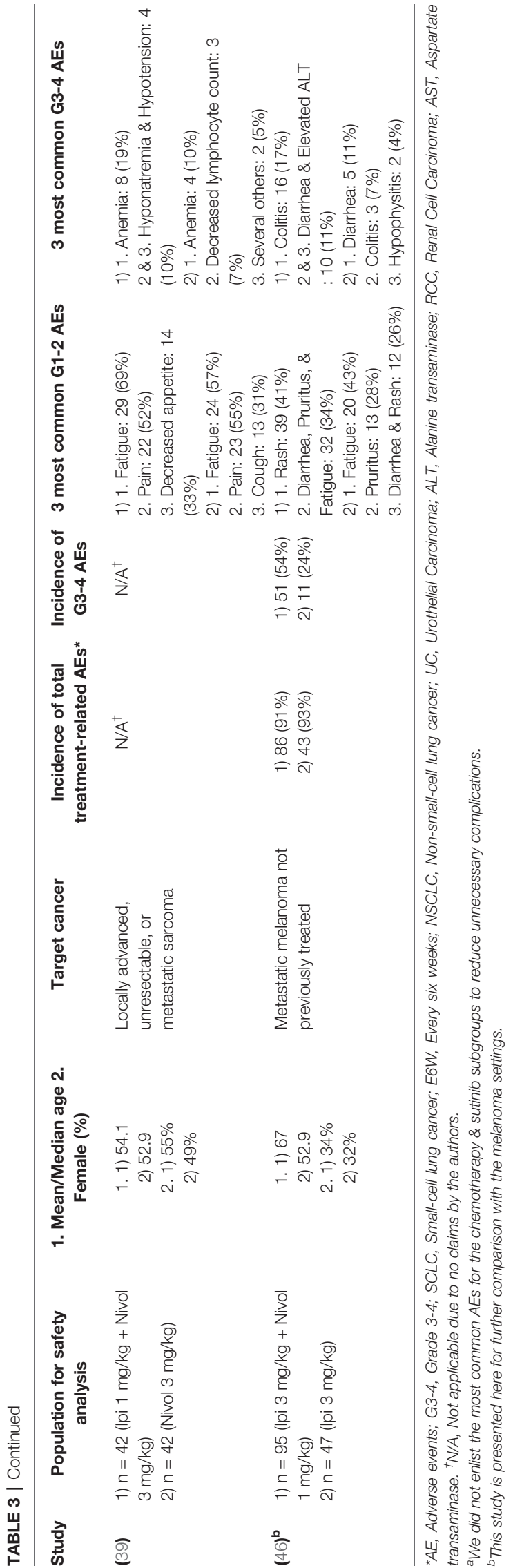

\section{Ipilimumab Versus Placebo in Combination Therapies}

Herein, we discuss irAEs data in the clinical trials investigating the ipilimumab versus placebo in combination therapies. Weber and colleagues have compared ipilimumab $3 \mathrm{mg} / \mathrm{kg}$ plus glycoprotein 100 melanoma antigen vaccine (gp100) (group A) and placebo plus gp100 (group B) (49). In this study, ipilimumab was administered up to four times every three weeks followed by the treatment continuation if the patients met the conditions. They found that overall treatment-related AEs, G3-4 AEs, overall irAEs, and G3-4 irAEs for group A $(\mathrm{n}=131)$ were $105(80.2 \%), 30(22.9 \%), 80$ (61.1\%), and $19(14.5 \%)$, respectively. For group B $(n=132)$ these numbers were $104(78.8 \%), 15$ (11.4\%), 42 (31.8\%), and $4(3 \%)$. Deaths related to the treatment were four in group A and two for group B. Most of the adverse events, including G3-4 symptoms, were reversible using vigilant monitoring and treatment.

In another study (50), the patients randomized into ipilimumab $10 \mathrm{mg} / \mathrm{kg}$ plus dacarbazine (group A) and placebo plus dacarbazine (group B), given in four treatment cycles every three weeks. Then both groups were received dacarbazine alone every three weeks four times. Maintenance therapy using ipilimumab or placebo every 12 weeks was also recommended for candidates. Data of 247 patients from group $A$ and 251 patients from group $B$ were used for the safety assessment. All grades of AEs and irAEs were reported as 244 (98.8\%) and 192 (77.7\%) for group A and 236 (94\%) and 96 (38.2\%) for group B. G3-4 treatment-related AEs were more common among group A [139 (56.3\%) versus 69 (27.5\%) $(\mathrm{P}<$ $0.001)]$. The number of patients with G3-4 irAEs was $103(41.7 \%)$ against 15 (6.0\%). No drug-related death was reported in group A, whereas one of the group B patients experienced death owing to gastrointestinal hemorrhage.

In another study, Robert et al. have demonstrated that seven patients who survived for at least five years following ipilimumab maintenance therapy have manifested grade 3 or 4 irAEs. Of five patients who were experienced irAEs of any grade, all had skin irAEs, two suffered from GI irAEs, two showed increased ALT or AST, and one had endocrine irAE. G3-4 irAEs were only observed in one patient and they were restricted to the skin (51).

The safety profile of the studies discussed in this group resembles the ones in the previous section. Although the total AEs remained similar, G3-4 AEs and irAEs were increased in the ipilimumab subgroup. Therefore, G3-4 irAEs surged to the highest level where ipilimumab was added to the treatment regimen. Furthermore, no study in both sections was reported a significant increase in the mortality rate of the patients receiving ipilimumab.

\section{Ipilimumab Dose Comparison}

Understanding and comparing the side effects of various doses of ipilimumab and will help the patients suffer less where higher AEs overshadow the clinical benefit. Ascierto and colleagues (52) have demonstrated that the overall adverse events and irAEs with any grade were $286 / 364(79 \%)$ and $269 / 364$ (74\%) of the patients in $10 \mathrm{mg} / \mathrm{kg}$ group, and 228/362 (63\%) and 197/362 (54\%) in 3 $\mathrm{mg} / \mathrm{kg}$ group, respectively. G3-4 irAEs were also seen in 110 (30\%) of the $10 \mathrm{mg} / \mathrm{kg}$ subgroup and $50(14 \%)$ of the $3 \mathrm{mg} / \mathrm{kg}$ arm. Diarrhea [10 mg/kg arm: 37 (10\%) vs. $3 \mathrm{mg} / \mathrm{kg}$ arm: 21 (6\%)], colitis [10 mg/kg arm: 19 (5\%) vs. $3 \mathrm{mg} / \mathrm{kg}$ arm: $9(2 \%)]$, 
and increased alanine aminotransferase $[10 \mathrm{mg} / \mathrm{kg}$ arm: 12 (3\%) vs. $3 \mathrm{mg} / \mathrm{kg}$ arm: 2 (1\%)] were accounted for the most common G3-4 treatment-related AEs. Death due to irAEs was noted in a patient in $3 \mathrm{mg} / \mathrm{kg}$ group owing to large intestinal perforation. In another study, overall irAEs were closer for $10 \mathrm{mg} / \mathrm{kg}$ [50 of 71 (70.4\%)] and $3 \mathrm{mg} / \mathrm{kg}$ [46 of $71(64.8 \%)$ ], where compared with $0.3 \mathrm{mg} / \mathrm{kg}$ arm [19 of 72 (26.4\%)]. No patient was developed G34 irAEs in the $0.3 \mathrm{mg} / \mathrm{kg}$ arm. However, five patients in the $3 \mathrm{mg} /$ $\mathrm{kg}$ subgroup and 18 in the $10 \mathrm{mg} / \mathrm{kg}$ arm were diagnosed with G3-4 irAEs. The most common observed irAEs were gastrointestinal (11 in the $10 \mathrm{mg} / \mathrm{kg}$ subgroup, two in the 3 $\mathrm{mg} / \mathrm{kg}$ ) (53). Following a survey on patients developing hypophysitis, Albarel et al. (54) have found that although 62 of the total 131 patients were received ipilimumab at a $3 \mathrm{mg} / \mathrm{kg}$ dosage, $11 / 15$ of the patients developing this condition were treated at a dosage of $10 \mathrm{mg} / \mathrm{kg}$. Interestingly, all 15 patients have suffered from at least one hormonal deficiency. While the number of tyrotroph, gonadotroph, and corticotroph deficiencies was similar (13, 12, and 11, respectively), no antidiuretic hormone $(\mathrm{ADH})$ deficiency (diabetes insipidus) was observed. This finding points out that $\mathrm{ADH}$ is probably not affected or much less negatively affected by ipilimumab therapy. Clinical symptoms were usually improved swiftly on high-dose glucocorticoids or by physiological replacement doses. Apart from corticotroph deficiency cases, hormonal deficiencies were also improved. In a study (55), ipilimumab was administered every three weeks to advanced melanoma adolescent patients aging 12-18. They have reported that one out of four patients receiving $3 \mathrm{mg} / \mathrm{kg}$ ipilimumab treatment was developed G3-4 irAEs, compared to 5 out of 8 patients receiving $10 \mathrm{mg} / \mathrm{kg}$ ipilimumab.

Overall, healthcare providers should be aware that patients treated with ipilimumab might develop irAEs. It is demonstrated that most irAEs can be reversible (56). Specific practical guidelines have been developed to help patients and practitioners to manage irAEs $(57,58)$. Systemic corticosteroids might be required in 35\% of the patients, and $10 \%$ may further need anti-TNF $\alpha$ for immune-suppression. These treatments could not alter overall survival or time to treatment failure (14). Endocrinopathies such as hypophysitis, although not frequent, might be too severe and practitioners may, therefore, seek long-term hormone replacement therapy (57).

Altogether, the overall evidence suggests that the safety profile might be independent or slightly dependent on underlying cancer (Tables 2 and 3). However, some studies may confront this claim and show variations related to different tumor types (31). For example, G3-4 hypophysitis might be more pronounced in melanoma settings $(46,47,54)$. Concurrently, a study on the patients with advanced or metastatic sarcoma

\section{REFERENCES}

1. Reiman JM, Kmieciak M, Manjili MH, Knutson KL. Tumor immunoediting and immunosculpting pathways to cancer progression. Semin Cancer Biol (2007) 17(4):275-87. doi: 10.1016/j.semcancer.2007.06.009

2. Hoos A, Britten A, Huber C, O'Donnell-Tormey J. A methodological framework to enhance the clinical success of cancer immunotherapy. Nat Biotechnol (2011) 29(10):867-70. doi: 10.1038/nbt.2000 receiving ipilimumab plus nivolumab (39) has shown a difference in other settings in which the patients received the same treatment (Table 3). Nevertheless, the lack of enough information on some conditions in the studies or random incidents might have resulted in these variations. Therefore, careful meta-analyses need to address this matter.

The pattern of AEs seems to be related more with the type of therapy (i.e., the dosage of ipilimumab and the type of concurrent therapies) $(39,50)$. Increasing the dosage may impose a higher risk of AEs while providing an overall advantage. Furthermore, the administration of other agents may increase the risk of specific types of AEs. An example is an excessive increase in alanine and aspartate aminotransferase (ALT and AST) where ipilimumab is co-administered with dacarbazine, a known hepatotoxic agent, in the metastatic melanoma settings (50). We suggest that evaluation of AEs should be conducted in every clinical trial in particular in the context of combination therapies.

\section{CONCLUSION}

Ipilimumab has shown promising results in many forms of advanced cancers evidenced by numerous trials. However, clinicians should always bear in mind that these benefits come at the cost of adverse events. Patients should always be informed about these side effects because their awareness might influence their trust and hope. Conversely, no awareness might result in their perplexity. We know that if the patients lose their trust in their treatment and attending physicians, they might lose their last chance of getting cured. Furthermore, when the signs and symptoms of adverse events start to manifest, timely management should be executed according to the approved guidelines and protocols. Further studies may require deciphering the hidden aspects of this area.

\section{AUTHOR CONTRIBUTIONS}

Conception and design of the paper were done by AK and HM. The initial draft was completed by AK. Editing and further drafts were done by HM. SA contributed to the critical discussion, visual design, and figure illustration. All authors contributed to the article and approved the submitted version.

\section{ACKNOWLEDGMENTS}

We would like to acknowledge Dr. Sanam Alilou for her sincere assistance in the preparation of the graphical abstract.

3. Allison JP, Krummel MF. The Yin and Yang of T cell costimulation. Science (1995) 270(5238):932-3. doi: 10.1126/science.270.5238.932

4. Krummel MF, Allison JP. CTLA-4 engagement inhibits IL-2 accumulation and cell cycle progression upon activation of resting T cells. J Exp Med (1996) 183(6):2533-40. doi: 10.1084/jem.183.6.2533

5. Peggs KS, Quezada SA, Korman AJ, Allison JP. Principles and use of antiCTLA4 antibody in human cancer immunotherapy. Curr Opin Immunol (2006) 18(2):206-13. doi: 10.1016/j.coi.2006.01.011 
6. Weber J. Ipilimumab: controversies in its development, utility and autoimmune adverse events. Cancer Immunol Immunother (2009) 58 (5):823-30. doi: 10.1007/s00262-008-0653-8

7. Juszczak A, Gupta A, Karavitaki N, Middleton MR, Grossman AB. Ipilimumab: a novel immunomodulating therapy causing autoimmune hypophysitis: a case report and review. Eur J Endocrinol (2012) 167(1):1-5. doi: 10.1530/EJE-12-0167

8. Fife BT, Bluestone JA. Control of peripheral T-cell tolerance and autoimmunity via the CTLA-4 and PD-1 pathways. Immunol Rev (2008) 224:166-82. doi: 10.1111/j.1600-065X.2008.00662.x

9. Teft WA, Kirchhof MG, Madrenas J. A molecular perspective of CTLA-4 function. Annu Rev Immunol (2006) 24:65-97. doi: 10.1146/ annurev.immunol.24.021605.090535

10. de Coana YP, Wolodarski M, Poschke I, Yoshimoto Y, Yang Y, Nystrom M, et al. Ipilimumab treatment decreases monocytic MDSCs and increases CD8 effector memory $\mathrm{T}$ cells in long-term survivors with advanced melanoma. Oncotarget (2017) 8(13):21539-53. doi: 10.18632/oncotarget.15368

11. Saleh K, Khalife-Saleh N, Kourie HR. Do immune-related adverse events correlate with response to immune checkpoint inhibitors? Immunotherapy (2019) 11(4):257-9. doi: 10.2217/imt-2018-0201

12. Downey SG, Klapper JA, Smith FO, Yang JC, Sherry RM, Royal RE, et al. Prognostic factors related to clinical response in patients with metastatic melanoma treated by CTL-associated antigen- 4 blockade. Clin Cancer Res (2007) 13(22 Pt 1):6681-8. doi: 10.1158/1078-0432.CCR-07-0187

13. Weber JS, O'Day S, Urba W, Powderly J, Nichol G, Yellin M, et al. Phase I/II study of ipilimumab for patients with metastatic melanoma. J Clin Oncol (2008) 26(36):5950-6. doi: 10.1200/JCO.2008.16.1927

14. Horvat TZ, Adel NG, Dang TO, Momtaz P, Postow MA, Callahan MK, et al. Immune-Related Adverse Events, Need for Systemic Immunosuppression, and Effects on Survival and Time to Treatment Failure in Patients With Melanoma Treated With Ipilimumab at Memorial Sloan Kettering Cancer Center. J Clin Oncol (2015) 33 (28):3193-8. doi: 10.1200/JCO.2015.60.8448

15. Arriola E, Wheater M, Galea I, Cross N, Maishman T, Hamid D, et al. Outcome and Biomarker Analysis from a Multicenter Phase 2 Study of Ipilimumab in Combination with Carboplatin and Etoposide as First-Line Therapy for Extensive-Stage SCLC. J Thorac Oncol (2016) 11(9):1511-21. doi: 10.1016/j.jtho.2016.05.028

16. Antonia SJ, Lopez-Martin JA, Bendell J, Ott PA, Taylor M, Eder JP, et al. Nivolumab alone and nivolumab plus ipilimumab in recurrent small-cell lung cancer (CheckMate 032): a multicentre, open-label, phase 1/2 trial. Lancet Oncol (2016) 17(7):883-95. doi: 10.1016/S1470-2045(16)30098-5

17. Reck M, Bondarenko I, Luft A, Serwatowski P, Barlesi F, Chacko R, et al. Ipilimumab in combination with paclitaxel and carboplatin as first-line therapy in extensive-disease-small-cell lung cancer: results from a randomized, double-blind, multicenter phase 2 trial. Ann Oncol (2013) 24 (1):75-83. doi: 10.1093/annonc/mds213

18. Reck M, Luft A, Szczesna A, Havel L, Kim SW, Akerley W, et al. Phase III Randomized Trial of Ipilimumab Plus Etoposide and Platinum Versus Placebo Plus Etoposide and Platinum in Extensive-Stage Small-Cell Lung Cancer. J Clin Oncol (2016) 34(31):3740-8. doi: 10.1200/JCO.2016.67.6601

19. Govindan R, Szczesna A, Ahn MJ, Schneider CP, Gonzalez Mella PF, Barlesi F, et al. Phase III Trial of Ipilimumab Combined With Paclitaxel and Carboplatin in Advanced Squamous Non-Small-Cell Lung Cancer. J Clin Oncol (2017) 35(30):3449-57. doi: 10.1200/JCO.2016.71.7629

20. Hellmann MD, Rizvi NA, Goldman JW, Gettinger SN, Borghaei H, Brahmer JR, et al. Nivolumab plus ipilimumab as first-line treatment for advanced non-smallcell lung cancer (CheckMate 012): results of an open-label, phase 1, multicohort study. Lancet Oncol (2017) 18(1):31-41. doi: 10.1016/S1470-2045(16)30624-6

21. Hellmann MD, Ciuleanu TE, Pluzanski A, Lee JS, Otterson GA, AudigierValette C, et al. Nivolumab plus Ipilimumab in Lung Cancer with a High Tumor Mutational Burden. N Engl J Med (2018) 378(22):2093-104. doi: 10.1056/NEJMoa1801946

22. Lynch TJ, Bondarenko I, Luft A, Serwatowski P, Barlesi F, Chacko R, et al. Ipilimumab in combination with paclitaxel and carboplatin as first-line treatment in stage IIIB/IV non-small-cell lung cancer: results from a randomized, double-blind, multicenter phase II study. J Clin Oncol (2012) 30(17):2046-54. doi: 10.1200/JCO.2011.38.4032
23. Horinouchi H, Yamamoto N, Fujiwara Y, Sekine I, Nokihara H, Kubota K, et al. Phase I study of ipilimumab in phased combination with paclitaxel and carboplatin in Japanese patients with non-small-cell lung cancer. Invest New Drugs (2015) 33(4):881-9. doi: 10.1007/s10637-015-0243-5

24. Yang CJ, McSherry F, Mayne NR, Wang X, Berry MF, Tong B, et al. Surgical Outcomes After Neoadjuvant Chemotherapy and Ipilimumab for Non-Small Cell Lung Cancer. Ann Thorac Surg (2018) 105(3):924-9. doi: 10.1016/ j.athoracsur.2017.09.030

25. Jochems C, Tucker JA, Tsang KY, Madan RA, Dahut WL, Liewehr DJ, et al. A combination trial of vaccine plus ipilimumab in metastatic castration-resistant prostate cancer patients: immune correlates. Cancer Immunol Immunother (2014) 63(4):407-18. doi: 10.1007/s00262-014-1524-0

26. Beer TM, Kwon ED, Drake CG, Fizazi K, Logothetis C, Gravis G, et al. Randomized, Double-Blind, Phase III Trial of Ipilimumab Versus Placebo in Asymptomatic or Minimally Symptomatic Patients With Metastatic Chemotherapy-Naive Castration-Resistant Prostate Cancer. J Clin Oncol (2017) 35(1):40-7. doi: 10.1200/JCO.2016.69.1584

27. Kwon ED, Drake CG, Scher HI, Fizazi K, Bossi A, van den Eertwegh AJ, et al. Ipilimumab versus placebo after radiotherapy in patients with metastatic castration-resistant prostate cancer that had progressed after docetaxel chemotherapy (CA184-043): a multicentre, randomised, double-blind, phase 3 trial. Lancet Oncol (2014) 15(7):700-12. doi: 10.1016/S1470-2045 (14)70189-5

28. Santegoets SJ, Stam AG, Lougheed SM, Gall H, Scholten PE, Reijm M, et al. T cell profiling reveals high CD4+CTLA- $4+\mathrm{T}$ cell frequency as dominant predictor for survival after prostate GVAX/ipilimumab treatment. Cancer Immunol Immunother (2013) 62(2):245-56. doi: 10.1007/s00262-012-1330-5

29. Slovin SF, Higano CS, Hamid O, Tejwani S, Harzstark A, Alumkal JJ, et al. Ipilimumab alone or in combination with radiotherapy in metastatic castration-resistant prostate cancer: results from an open-label, multicenter phase I/II study. Ann Oncol (2013) 24(7):1813-21. doi: 10.1093/annonc/ mdt107

30. Galsky MD, Wang H, Hahn NM, Twardowski P, Pal SK, Albany C, et al. Phase 2 Trial of Gemcitabine, Cisplatin, plus Ipilimumab in Patients with Metastatic Urothelial Cancer and Impact of DNA Damage Response Gene Mutations on Outcomes. Eur Urol (2018) 73(5):751-9. doi: 10.1016/j.eururo.2017.12.001

31. Sharma P, Siefker-Radtke A, de Braud F, Basso U, Calvo E, Bono P, et al. Nivolumab Alone and With Ipilimumab in Previously Treated Metastatic Urothelial Carcinoma: CheckMate 032 Nivolumab $1 \mathrm{mg} / \mathrm{kg}$ Plus Ipilimumab $3 \mathrm{mg} / \mathrm{kg}$ Expansion Cohort Results. J Clin Oncol (2019) 37(19):1608-16. doi: 10.1200/JCO.19.00538

32. Hammers HJ, Plimack ER, Infante JR, Rini BI, McDermott DF, Lewis LD, et al. Safety and Efficacy of Nivolumab in Combination With Ipilimumab in Metastatic Renal Cell Carcinoma: The CheckMate 016 Study. J Clin Oncol (2017) 35(34):3851-8. doi: 10.1200/JCO.2016.72.1985

33. Motzer RJ, Tannir NM, McDermott DF, Aren Frontera O, Melichar B, Choueiri TK, et al. Nivolumab plus Ipilimumab versus Sunitinib in Advanced Renal-Cell Carcinoma. N Engl J Med (2018) 378(14):1277-90. doi: 10.1056/NEJMoa1712126

34. Yang JC, Hughes M, Kammula U, Royal R, Sherry RM, Topalian SL, et al. Ipilimumab (anti-CTLA4 antibody) causes regression of metastatic renal cell cancer associated with enteritis and hypophysitis. J Immunother (2007) 30 (8):825-30. doi: 10.1097/CJI.0b013e318156e47e

35. Bang YJ, Cho JY, Kim YH, Kim JW, Di Bartolomeo M, Ajani JA, et al. Efficacy of Sequential Ipilimumab Monotherapy versus Best Supportive Care for Unresectable Locally Advanced/Metastatic Gastric or Gastroesophageal Junction Cancer. Clin Cancer Res (2017) 23(19):5671-8. doi: 10.1158/10780432.CCR-17-0025

36. Le DT, Lutz E, Uram JN, Sugar EA, Onners B, Solt S, et al. Evaluation of ipilimumab in combination with allogeneic pancreatic tumor cells transfected with a GM-CSF gene in previously treated pancreatic cancer. J Immunother (2013) 36(7):382-9. doi: 10.1097/CJI.0b013e31829fb7a2

37. Royal RE, Levy C, Turner K, Mathur A, Hughes M, Kammula US, et al. Phase 2 trial of single agent Ipilimumab (anti-CTLA-4) for locally advanced or metastatic pancreatic adenocarcinoma. J Immunother (2010) 33(8):828-33. doi: 10.1097/CJI.0b013e3181eec14c

38. Merchant MS, Wright M, Baird K, Wexler LH, Rodriguez-Galindo C, Bernstein D, et al. Phase I Clinical Trial of Ipilimumab in Pediatric Patients 
with Advanced Solid Tumors. Clin Cancer Res (2016) 22(6):1364-70. doi: 10.1158/1078-0432.CCR-15-0491

39. D’Angelo SP, Mahoney MR, Van Tine BA, Atkins J, Milhem MM, Jahagirdar BN, et al. Nivolumab with or without ipilimumab treatment for metastatic sarcoma (Alliance A091401): two open-label, non-comparative, randomised, phase 2 trials. Lancet Oncol (2018) 19(3):416-26. doi: 10.1016/S1470-2045(18) 30006-8

40. Ansell SM, Hurvitz SA, Koenig PA, LaPlant BR, Kabat BF, Fernando D, et al. Phase I study of ipilimumab, an anti-CTLA-4 monoclonal antibody, in patients with relapsed and refractory B-cell non-Hodgkin lymphoma. Clin Cancer Res (2009) 15(20):6446-53. doi: 10.1158/1078-0432.CCR-09-1339

41. Davids MS, Kim HT, Bachireddy p, Costello C, Liguori R, Savell A, et al. Ipilimumab for Patients with Relapse after Allogeneic Transplantation. N Engl J Med (2016) 375(2):143-53. doi: 10.1056/NEJMoa1601202

42. Bashey A, Medina B, Corringham S, Pasek M, Carrier E, Vrooman L, et al. CTLA4 blockade with ipilimumab to treat relapse of malignancy after allogeneic hematopoietic cell transplantation. Blood (2009) 113(7):1581-8. doi: 10.1182/blood-2008-07-168468

43. Sakurai J, Ohata J, Saito K, Miyajima H, Hirano T, Kohsaka T, et al. Blockade of CTLA-4 signals inhibits Th2-mediated murine chronic graft-versus-host disease by an enhanced expansion of regulatory CD8+ T cells. J Immunol (2000) 164 (2):664-9. doi: 10.4049/jimmunol.164.2.664

44. Hodi FS, O'Day SJ, McDermott DF, Weber RW, Sosman JA, Haanen JB, et al. Improved survival with ipilimumab in patients with metastatic melanoma. $N$ Engl J Med (2010) 363(8):711-23. doi: 10.1056/NEJMoa1003466

45. Ferris RL, Blumenschein G Jr., Fayette J, Guigay J, Colevas AD, Licitra RL, et al. Nivolumab for Recurrent Squamous-Cell Carcinoma of the Head and Neck. N Engl J Med (2016) 375(19):1856-67. doi: 10.1056/NEJMoa1602252

46. Postow MA, Chesney J, Pavlick AC, Robert C, Grossmann K, McDermott D, et al. Nivolumab and ipilimumab versus ipilimumab in untreated melanoma. N Engl J Med (2015) 372(21):2006-17. doi: 10.1056/NEJMoa1414428

47. Eggermont AM, Chiarion-Sileni V, Grob JJ, Dummer R, Wolchok JD, Schmidt $\mathrm{H}$, et al. Adjuvant ipilimumab versus placebo after complete resection of high-risk stage III melanoma (EORTC 18071): a randomised, double-blind, phase 3 trial. Lancet Oncol (2015) 16(5):522-30. doi: 10.1016/ S1470-2045(15)70122-1

48. Coens C, Suciu S, Chiarion-Sileni V, Grob JJ, Dummer R, Wolchok JD, et al. Health-related quality of life with adjuvant ipilimumab versus placebo after complete resection of high-risk stage III melanoma (EORTC 18071): secondary outcomes of a multinational, randomised, double-blind, phase 3 trial. Lancet Oncol (2017) 18(3):393-403. doi: 10.1016/S1470-2045(17)30015-3

49. Weber JS, Dummer R, de Pril V, Lebbe C, Hodi FS. Patterns of onset and resolution of immune-related adverse events of special interest with ipilimumab: detailed safety analysis from a phase 3 trial in patients with advanced melanoma. Cancer (2013) 119(9):1675-82. doi: 10.1002/cncr.27969

50. Robert C, Thomas L, Bondarenko I, O'Day C, Weber J, Garbe C, et al. Ipilimumab plus dacarbazine for previously untreated metastatic melanoma. N Engl J Med (2011) 364(26):2517-26. doi: 10.1056/ NEJMoa1104621

51. Maio M, Grob JJ, Aamdal S, Bondarenko I, Robert C, Thomas M, et al. Fiveyear survival rates for treatment-naive patients with advanced melanoma who received ipilimumab plus dacarbazine in a phase III trial. J Clin Oncol (2015) 33(10):1191-6. doi: 10.1200/JCO.2014.56.6018

52. Ascierto PA, Del Vecchio M, Robert C, Mackiewicz A, Chiarion-Sileni V, Arance A, et al. Ipilimumab $10 \mathrm{mg} / \mathrm{kg}$ versus ipilimumab $3 \mathrm{mg} / \mathrm{kg}$ in patients with unresectable or metastatic melanoma: a randomised, double-blind, multicentre, phase 3 trial. Lancet Oncol (2017) 18(5):611-22. doi: 10.1016/ S1470-2045(17)30231-0

53. Wolchok JD, Neyns B, Linette G, Negrier S, Lutzky J, Thomas L, et al. Ipilimumab monotherapy in patients with pretreated advanced melanoma: a randomised, double-blind, multicentre, phase 2, doseranging study. Lancet Oncol (2010) 11(2):155-64. doi: 10.1016/S14702045(09)70334-1

54. Albarel F, Gaudy C, Castinetti F, Carre T, Morange I, Conte-Devolx B, et al. Long-term follow-up of ipilimumab-induced hypophysitis, a common adverse event of the anti-CTLA-4 antibody in melanoma. Eur J Endocrinol (2015) 172 (2):195-204. doi: 10.1530/EJE-14-0845

55. Geoerger B, Bergeron C, Gore L, Sender L, Dunkel IJ, Herzog C, et al. Phase II study of ipilimumab in adolescents with unresectable stage III or IV malignant melanoma. Eur J Cancer (2017) 86:358-63. doi: 10.1016/ j.ejca.2017.09.032

56. Wolchok JD, Hodi FS, Weber JS, Allison JP, Urba WJ, Robert C, et al. Development of ipilimumab: a novel immunotherapeutic approach for the treatment of advanced melanoma. Ann N Y Acad Sci (2013) 1291:1-13. doi: 10.1111 /nyas. 12180

57. Weber JS, Kahler KC, Hauschild A. Management of immune-related adverse events and kinetics of response with ipilimumab. J Clin Oncol (2012) 30 (21):2691-7. doi: 10.1200/JCO.2012.41.6750

58. Kaehler KC, Piel S, Livingstone E, Schilling B, Hauschild A, Schadendorf KC, et al. Update on immunologic therapy with anti-CTLA-4 antibodies in melanoma: identification of clinical and biological response patterns, immune-related adverse events, and their management. Semin Oncol (2010) 37(5):485-98. doi: 10.1053/j.seminoncol.2010.09.003

Conflict of Interest: The authors declare that the research was conducted in the absence of any commercial or financial relationships that could be construed as a potential conflict of interest.

Copyright (C) 2021 Karimi, Alilou and Mirzaei. This is an open-access article distributed under the terms of the Creative Commons Attribution License (CC BY). The use, distribution or reproduction in other forums is permitted, provided the original author(s) and the copyright owner(s) are credited and that the original publication in this journal is cited, in accordance with accepted academic practice. No use, distribution or reproduction is permitted which does not comply with these terms. 